\title{
Meaning, Truth, and Physics
}

\author{
László E. Szabó \\ Department of Logic, Institute of Philosophy \\ Eötvös Loránd University Budapest
}

\begin{abstract}
A physical theory is a partially interpreted axiomatic formal system $(L, S)$, where $L$ is a formal language with some logical, mathematical and physical axioms, and with some derivation rules, and the semantics $S$ is a relationship between the formulas of $L$ and some states of affairs in the physical world. In our ordinary discourse, the formal system $L$ is regarded as an abstract object or structure, the semantics $S$ as something which involves the mental/conceptual realm. This view is of course incompatible with physicalism. How can physical theory be accommodated in a purely physical ontology? The aim of this paper is to outline an account for meaning and truth of physical theory, within the philosophical framework spanned by three doctrines: physicalism, empiricism, and the formalist philosophy of mathematics.
\end{abstract}

\section{Introduction}

1. All the forthcoming considerations will be based on the following three philosophical premises.

Physicalism: everything is physical; all facts supervene on, or are necessitated by, the physical facts.

Empiricism: $\quad$ genuine information about the world can be acquired only by a posteriori means.

Formalism: $\quad$ logic and mathematics are thought of as statements about manipulations with meaningless symbols.

I won't argue for these doctrines in this paper - they are legitimate philosophical positions. I take them as initial premises. Rather, I will discuss a few radical 
consequences of them, concerning the fundamental nature of logic and mathematics, and the structure of physical theories. Nevertheless, it must be mentioned that the ontological doctrine of physicalism is of primary importance, which, in some sense, implies both formalism and empiricism. The first implication is rather trivial. Physicalism denies the existence of mental and abstract entities; consequently, there is no room left for any kind of platonism or mentalism in the philosophy of logic and mathematics. Therefore, formalism - more precisely, what we will call physco-formalism - seems to be the only account for logic and mathematics that can be compatible with physicalism. Regarding the second implication, we will see in point $\mathbf{1 0}$ that empiricism will be a consequence of our physico-formalist account of the semantics of physical theory.

In the first section I will outline the basic ideas of physico-formalist philosophy of mathematics. In the second section, combining the physico-formalist approach to formal system with an intuition we can learn from Gödel's proof of incompleteness theorem, I will sketch a physicalist-formalist-empiricist - or shortly, physicalist - theory of meaning and truth with respect to physical theories.

\section{Physico-formalist philosophy of mathematics}

2. Physico-formalist interpretation of mathematics (Szabó 2003; 2012) is a reflection to the following fundamental problem: If physicalism is true, then the logical/mathematical facts must be necessitated by the physical facts of the world. This raises the question: How can the logical and mathematical facts be accommodated in a purely physical ontology? In order to answer this question, first we need to clarify: What is it that has to be accounted for within a physicalist ontology? What are the logical/mathematical facts?

Our starting point is the formalist thesis. In Hilbert's famous formulation it says:

The formalist thesis: "Mathematics is a game played according to certain simple rules with meaningless marks." (Bell 1951, 38)

In other words, a mathematical statement/fact/truth is like " $\Sigma \vdash A$ " - with single turnstile, it must be emphasized. Note that this claim is more radical than, as sometimes called, "if-thenism", according to which the truth of $A$ is based on the assumption that the premises in $\Sigma$ are true. According to the formalist view, neither $A$ nor the elements of $\Sigma$ are statements, which could be true or false. They are just strings, formulas of the formal system in question. Derivation is not a truth preserving "if-then"-type reasoning as traditionally taken, but a mechanistic operation with meaningless strings of symbols. 


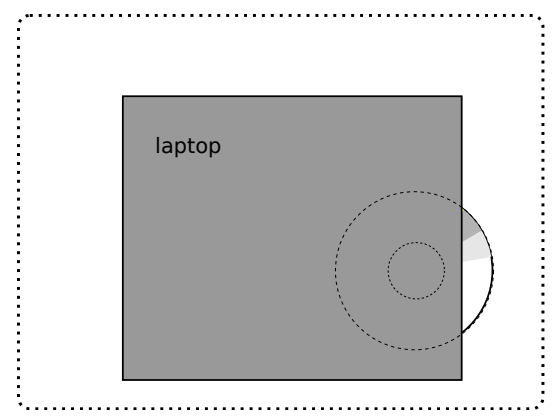

Figure 1: A formal system represented in a laptop with an inserted $C D$. The $C D$ contains a program making the computer to list the theorems of the formal system

3. This is the point where physico-formalist philosophy of mathematics starts. We turn to the following question: Where are the states of affairs located in the ontological picture of the world that make propositions like " $\Sigma \vdash A$ " true or false? The physico-formalist answer is this:

The physico-formalist thesis: A formal system should be regarded as a physical system which consists of signs and derivational mechanisms embodied in concrete physical objects, concrete physical configurations, and concrete physical processes.

Therefore, a " $\Sigma \vdash A$ "-type mathematical proposition expresses an objective fact of the formal system as a particular portion of the physical world.

One can argue for this physicalist account of formal system in three steps. (For more detailed argumentation see Szabó 2012).

(I) A formal systems can be represented in a physical system. Imagine a laptop with an inserted CD (Fig. 1). The CD contains a program completely determining the laptop's behavior: in some order, the computer lists the theorems (together with the proofs) of a formal system. I think, it is commonly accepted to say that in the "computer + CD" system we have "a physical representation of the formal system" in question. In this representation, " $\Sigma \vdash A$ " (that is, formula $A$ is printed) becomes a fact of the physical reality inside the dotted line.

(II) All mathematical facts can be thought of as a physical fact in some physical representation. It is because, in Curry's words, "in order to think of a formal system at all we must think of it as represented somehow". (Curry 1951, 30) This is in agreement with the widely accepted view in the recent literature 
on physical computation that "it is only physical objects, such as computers or human brains, that ever give us glimpses of the abstract world of mathematics". (Deutsch et al. 2000, 265)

(III) Actually, there is nothing to be "represented"; there is nothing beyond the flesh and blood formal systems. Consider the context of the above quoted sentence of Curry:

[A]lthough a formal system may be represented in various ways, yet the theorems derived according to the specifications of the primitive frame remain true without regard to changes in representation. There is, therefore, a sense in which the primitive frame defines a formal system as a unique object of thought. This does not mean that there is a hypostatized entity called a formal system which exists independently of any representation. On the contrary, in order to think of a formal system at all we must think of it as represented somehow. But when we think of it as formal system we abstract from all properties peculiar to the representation. (Curry 1951, 30)

But, what does such an "abstraction" actually mean? What do we obtain if we abstract from some unimportant, peculiar properties of a physical system $L$ constituting a "physical representation of a formal system"? In order to think of this abstraction at all, in order to differentiate the important and unimportant features of the physical system $L$, and to change from a more detailed description of the system to a less detailed one, we must have a physical theory $(M, S)$ in the sense of the definition of physical theory in the next section - describing the physical system $L$ in question. However, the formal system $M$ also is "represented somehow", in Curry's words; it is another flesh and blood formal system. That is to say, the whole abstraction - the result of the abstraction included - is contained in another flesh and blood formal system $M$. So, instead of obtaining a non-physical "abstract formal system" we remain to have flesh and blood formal systems.

Another way to think of this abstraction is to consider what is common to the different "physical representations", that is, to describe the common features of different flesh and blood formal systems $L_{1}, L_{2}, \ldots L_{n}$. But, to describe the common features of physical systems $L_{1}, L_{2}, \ldots L_{n}$ we have to have a physical theory ( $M, S$ ) which is capable of describing all $L_{1}, L_{2}, \ldots L_{n}$ together (Fig. 2). Only in a suitable formal system $M$ it is meaningful to talk about similarity or isomorphism between the structures describing $L_{1}, L_{2}, \ldots L_{n}$, and about the equivalence class of these structures, which could be regarded as an "abstract 


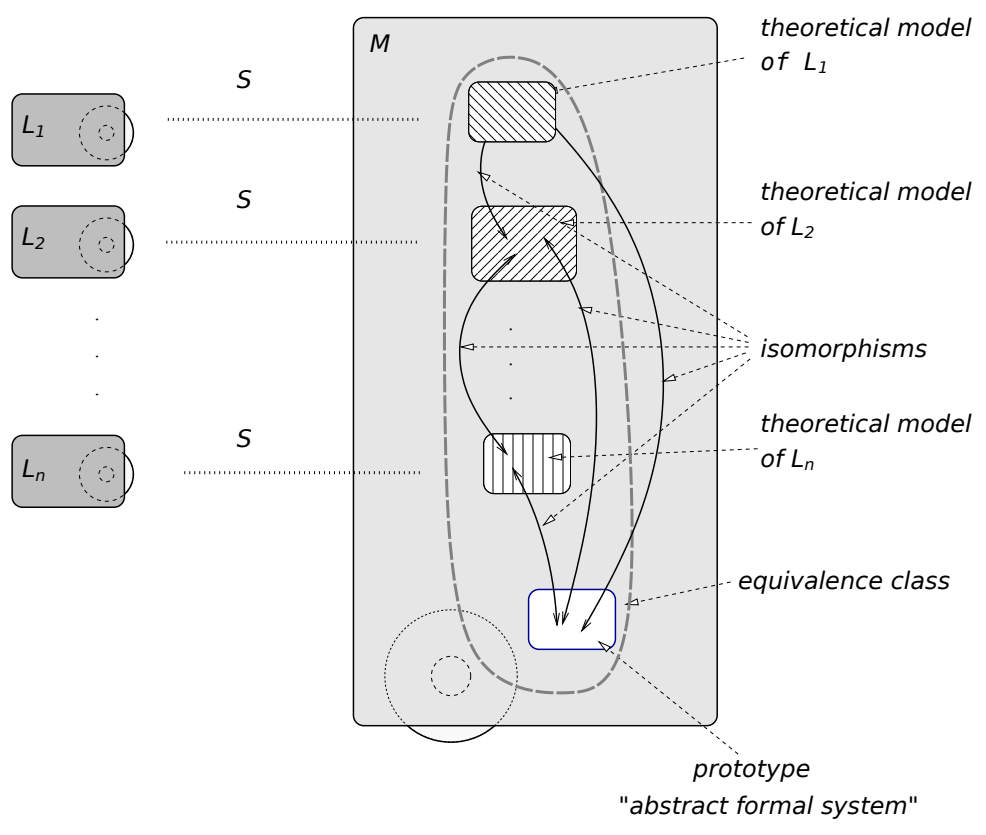

Figure 2: To describe the common features of physical systems $L_{1}, L_{2}, \ldots L_{n}$ we have to have a physical theory $(M, S)$ which is capable of describing all $L_{1}, L_{2}, \ldots L_{n}$ together

formal system". But, all these objects live in the formal system $M$ which also is "represented somehow", that is, in a formal system existing in the physical world.

Thus, abstraction, properly understood, does not lead out of the physical realm. It does not produce "abstract formal systems" over and above the physically existing "representations". Actually, there is nothing to be "represented"; there is nothing beyond the flesh and blood formal systems. That is to say, a formal system "as formal system" is a part of the physical reality. Consequently, any statement about a formal system - including a statement like " $\Sigma \vdash A$ " - is a statement of a physical fact; and it has exactly the same epistemological status as any other statements about the physical world. ${ }^{1}$

\footnotetext{
${ }^{1}$ Let me emphasize the distinction between the physico-formalist interpretation of mathematics and the so called immanent or physical realism in the sense of Mill. For example, ' $a^{2}+b^{2}=c^{2}$, is a theorem of Euclidean geometry, \{Euclidean $\} \vdash a^{2}+b^{2}=c^{2}$. According to immanent realism, ' $a^{2}+b^{2}=c^{2}$ ' reflects some general truth about the real triangles in the physical world. Or at least, in the sense of the structuralist version of immanent realism, the axiomatic theory "Euclidean geometry", as a whole, expresses some true structural property of the physical world; something
} 
4. There are far-reaching consequences of the above considerations concerning an old problem of empiricism. In Ayer's words:

Where the empiricist does encounter difficulty is in connection with the truths of formal logic and mathematics. For whereas a scientific generalization is readily admitted to be fallible, the truths of mathematics and logic appear to everyone to be necessary and certain. But if empiricism is correct no proposition which has a factual content can be necessary or certain. Accordingly the empiricist must deal with the truths of logic and mathematics in one of the following ways: he must say either that they are not necessary truths, in which case he must account for the universal conviction that they are; or he must say that they have no factual content, and then he must explain how a proposition which is empty of all factual content can be true and useful and surprising. ...

If neither of these courses proves satisfactory, we shall be obliged to give way to rationalism. We shall be obliged to admit that there are some truths about the world which we can know independently of experience; ... (Ayer 1952, 72.)

The physico-formalist approach resolves this difficulty in the following way:

1. Logical and mathematical truths express objective (mind independent) facts of a particular part of the physical world, namely, the facts of the formal systems themselves. As such, they are synthetic, a posteriori, not necessary, and not certain; they are fallible.

2. But they have contingent factual content, as any similar scientific assertion, so they "can be true and useful and surprising". The logical and mathematical facts can be discovered, like any other facts of nature, just like a fact about a plastic molecule, or other artifact.

3. The fact that the flesh and blood formal systems usually are simple physical systems of relatively stable behavior, like a clockwork, and that the knowledge of logical and mathematical truths does not require observations of the physical world external to the formal systems explains the universal illusion that logical and mathematical truths are necessary, certain and $a$ priori.

about the congruence of rigid bodies, or the likes. The physico-formalist theory also claims that \{Euclidean $\vdash \vdash a^{2}+b^{2}=c^{2}$ expresses something in the physical world. But this something has nothing to do with the physical triangles and rigid bodies. \{Euclidean\} $\vdash a^{2}+b^{2}=c^{2}$ expresses a property of the formal system "Euclidean geometry", a property of the physical system consisting of the signs and the derivation mechanisms. 
Thus, empiricism is not challenged by the alleged necessary truths delivered by logical and mathematical reasoning. On the contrary, consequent physicalism can resolve the long-standing debate surrounding the truth-of-reasoning versus truth-of-facts dichotomy. Logical and mathematical truths are nothing but knowledge obtained from experience with respect to particular physical systems, the formal systems themselves. Since logical and mathematical derivations are "reasoning" par excellence, one must conclude that reasoning does not deliver to us necessary truths. Logical and mathematical reasoning is, if you like, an experiment with a flesh and blood formal system. Therefore, we must draw the epistemological conclusion: There is no higher degree of certainty than available from experience.

5. Formal systems are man-maid physical objects. As such, they are not eternal and not readily given to us. Nevertheless, it is of course not true that anything can be created with arbitrary properties. The objective features of physical reality predetermine what can be created and what cannot. For example, even if we assume that there are no polyvinyl chloride molecules in the universe, except the ones created by mankind, the laws of nature admit the possibility of their existence and predetermine their properties. Similarly, the laws of nature predetermine what kinds of formal system can exist. In this sense, the logical and mathematical facts are eternal and independent from us. They are contingent as much as the laws of nature are contingent. In other words, Paracelsus' God can make "a triangle with four sides" exactly as well as "an ass with three tails". ${ }^{2}$

\section{Physical theory}

6. Following Carnap, a physical theory can be considered as a partially interpreted formal system, $(L, S)$, providing a description of a certain part of physical reality, $U$. In general, the system of axioms of $L$ contains some logical axioms and the derivation rules (ideally, the first-order predicate calculus with identity), the axioms of some mathematical theories, and, of course, some physical

\footnotetext{
${ }^{2}$ Notice that this claim of contingency of logical and mathematical facts is quite different from what we call mathematical contingentism in the debates about contingency vs. necessity of the existence of mathematical entities (e.g. Field 1993; Colyvan 2000; Miller 2012). In the physico-formalist approach a formula of a formal system carries no meaning; Platonic, fictional, whatsoever. The meaningful statements of logic and mathematics, which can be true or false, are of form " $\Sigma \vdash A$ ". They do refer, indeed, to real facts of a real object: the physically existing formal system. This object, as we will see in the next section, is indeed indispensable for our physical theories. (Cf. Colyvan 2004)
} 
axioms. (The distinction between "logical", "mathematical", and "physical" are rather terminological/traditional than essential.) The semantics $S$ is understood as a kind of correspondence between (some of) the formulas of $L$ and the states of affairs in $U$. Consider an interpreted formula $A$ in $L$. One has to distinguish between the following two notions:

1. A is a theorem in $L, L \vdash A$.

2. A is true, that is, according to the semantics $S, A$ refers to a state of affairs in $U$, which is in fact the case.

For example, 'The electric field strength of a point charge is $\frac{k Q}{r^{2}}$ is a theorem of Maxwell's electrodynamics - one can derive it from the Maxwell equations. On the other hand, according to the semantics relating the symbols of the Maxwell theory to the empirical terms, this sentence corresponds to an empirically observable fact of the physical reality.

A physical theory is considered to be true if these two things are in harmony: if $A$ is a prediction of the theory, $L \vdash A$, then it corresponds to a state of affairs which is indeed the case in $U$. By and large, this is the standard picture of a physical theory.

7. Let us turn to the ontological issues. It is perhaps not far from the truth to say that the standard view regards the formal system $L$ as an abstract object or structure, the semantics $S$ as something which involves the mental/conceptual realm, and, of course, $U$ as a part of the physical world. This view is of course incompatible with physicalism. How can physical theory be accommodated in a purely physical ontology? The $L$-part is already solved by the physico-formalist interpretation of formal system and logical/mathematical fact. But, how can the physicalist account for meaning and truth? Again, first we need to clarify what it is that has to be accounted for; we need a definition of semantic relationship between formulas of a formal system and states of affairs in the physical world.

The definition will be based on the intuition we can learn from Gödel's construction of representation of the meta-arithmetic facts in Peano arithmetic, in the preparation of the first incompleteness theorem (e.g. Crossley et al. 1990, 62). Let $\operatorname{Pr}(x, y)$ denote the meta-arithmetic fact that the formula-sequence of Gödel number $x$ constitutes a proof of the formula of Gödel number $y$. According to Gödel's construction, every such meta-arithmetic fact $\operatorname{Pr}(x, y)$ is represented with a PA formula $R(x, y)$ if the following condition is met: For all Gödel numbers $(x, y)$,

$$
\begin{array}{r}
\text { if } \operatorname{Pr}(x, y) \text { is the case then } \quad \Sigma_{\mathrm{PA}} \vdash R(x, y) \\
\text { if } \operatorname{Pr}(x, y) \text { is not the case } \quad \text { then } \quad \Sigma_{\mathrm{PA}} \vdash \neg R(x, y)
\end{array}
$$



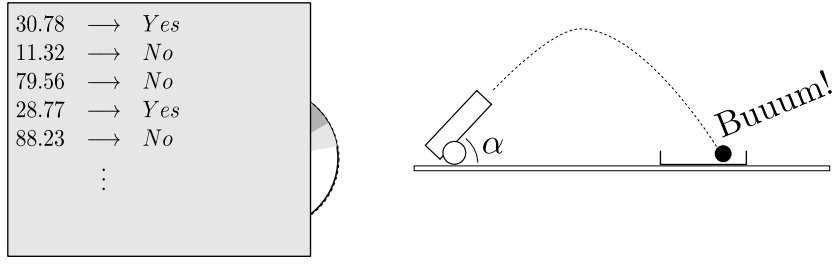

Figure 3: Imagine a computer programmed such that entering a random number $z \in(0,90)$ it calculates whether the bullet will land in the plate or not if the angle of inclination is $\%$. At the same time we perform the experiment with a randomly chosen angle of inclination $\alpha \in(0,90)$.

where $\Sigma_{\mathrm{PA}}$ denotes the axioms of Peano arithmetic.

Mutatis mutandis: one is entitled to say that a formula $A$ represents or means a state of affairs $a$ in $U$, if the following two conditions are met:

(a) There exist a family $\left\{A_{\lambda}\right\}_{\lambda}$ of formulas in $L$ and a family $\left\{a_{\lambda}\right\}_{\lambda}$ of states of affairs in $U$, such that $A=A_{\lambda_{0}}$ and $a=a_{\lambda_{0}}$ for some $\lambda_{0}$.

For all $\lambda$,

$$
\begin{aligned}
& \text { if } a_{\lambda} \text { is the case in } U \text { then } \Sigma \vdash A_{\lambda} \\
& \text { if } a_{\lambda} \text { is not the case in } U \text { then } \quad \Sigma \vdash \neg A_{\lambda}
\end{aligned}
$$

8. Turning to the ontological issues, it must be clear that $a_{\lambda}$ - as a symbol in the meta-language we use to describe the semantic relationship - stands for a state of affairs, a configuration of the physical world. It is not a linguistic object, it is not a "sentence of the theory expressing the state of affairs" in question. It is $A_{\lambda}$ that is a linguistic object, a formula of $L$, that can refer to $a_{\lambda}$, given that condition (b) holds.

According to the physico-formalist approach " $\Sigma \vdash A_{\lambda}$ " and " $\Sigma \vdash \neg A_{\lambda}$ ", respectively, are states of affairs in the physical world, facts of the flesh and blood formal system $L$. Thus, what we observe in condition (b) is a kind of regularity or correlation between physical facts of two parts of the physical world, $L$ and $U$. More precisely, it should be a real correlation. To explain what "real" means here, let me give a simple example. Imagine a computer programmed such that entering a random number $z \in(0,90)$ it calculates by applying some laws of physics whether the bullet shot from the gun will land in the plate or not if the angle of inclination is $z$ (Fig. 3). At the same time we perform the experiment 


\begin{tabular}{|c|c|c|c|c|}
\hline$\alpha$ & Outcome & & $z$ & Result \\
\hline \hline 88.23 & - & & 30.78 & Yes \\
\hline 79.56 & - & & 11.32 & No \\
\hline 30.78 & Buuum! & & 79.56 & No \\
\hline 11.32 & - & & 28.77 & Yes \\
\hline 28.77 & Buuum! & & 88.23 & No \\
\hline 29.02 & Buuum! & & 62.25 & No \\
\hline 62.25 & - & & 34.21 & Yes \\
\hline 36.54 & Buuum! & 29.02 & Yes \\
\hline 34.21 & Buuum! & & 36.54 & Yes \\
\hline
\end{tabular}

Table 1: Pairing by simultaneity

\begin{tabular}{|c|c|c|c|c|}
\hline$\alpha$ & Outcome & & $z$ & Result \\
\hline \hline 88.23 & - & & 11.32 & No \\
\hline 79.56 & - & & 79.56 & No \\
\hline 30.78 & Buuum! & & 30.78 & Yes \\
\hline 11.32 & - & & 88.23 & No \\
\hline 28.77 & Buuum! & & 28.77 & Yes \\
\hline 29.02 & Buuum! & 34.21 & Yes \\
\hline 62.25 & - & & 62.25 & No \\
\hline 36.54 & Buuum! & 29.02 & Yes \\
\hline 34.21 & Buuum! & & 36.54 & Yes \\
\hline
\end{tabular}

Table 2: A conceived pairing

with a randomly chosen angle of inclination $\alpha \in(0,90)$. Now, imagine a laboratory record as in Table 1 . In this table, the pairing between the facts of the computer and the facts of the experiment was based on simultaneity. It is based on a real physical circumstance establishing a real conjunctive relation between the elements of the two families. There is no correlation; condition (b) is not satisfied.

One can however conceive another parametrization of the two families as is shown in Table 2, according to which condition (b) is satisfied. Are we entitled to say that the computer makes real correct predictions? That a given "Yes" is a prediction of "Buuum!"? Of course not, because this parametrization is only a conceived one; it does not establish a real conjunctive relationship.

Finally, compare this with the normal situation when the pairing is based on the angle of inclination (Table 3). This parametrization is based on a real phys- 


\begin{tabular}{|c|c|c|c|c|}
\hline$\alpha$ & Outcome & & $z$ & Result \\
\hline \hline 11.32 & - & & 11.32 & No \\
\hline 28.77 & Buuum! & & 28.77 & Yes \\
\hline 29.02 & Buuum! & & 29.02 & Yes \\
\hline 30.78 & Buuum! & & 30.78 & Yes \\
\hline 34.21 & Buuum! & & 34.21 & Yes \\
\hline 36.54 & Buuum! & & 36.54 & Yes \\
\hline 62.25 & - & & 62.25 & No \\
\hline 79.56 & - & & 79.56 & No \\
\hline 88.23 & - & & 88.23 & No \\
\hline
\end{tabular}

Table 3: The normal pairing by the angle of inclination

ical circumstance establishing real conjunctive relation between the elements of the two families. There is maximal correlation; condition (b) is satisfied.

The upshot is that parameter $\lambda$ in conditions (a) and (b) is not completely arbitrary $^{3}$; not simply an "abstract" or conceived parametrization. It must have a physical realization, establishing a real conjunctive relationship between $a_{\lambda}$ and $A_{\lambda}$, by which condition (b) expresses a real correlation between physical facts of $L$ and physical facts of $U$.

With this strengthening all elements of semantic relationship become accommodated in the physical world.

9. A few important remarks are in order.

1. As we have seen, to be a meaning-carrier is not simply a matter of convention or definition or declaration. Semantics is not an arbitrary assignment of states of affairs of the world to linguistic elements of the theory.

2. It is pointless to talk about the meaning of an isolated formula of the theory. (Semantic holism) It is not only because of condition (a), but also because in condition (b) a big part of the axiomatic system can be involved.

3. It must be recognized that condition (b) is nothing but the necessary and sufficient condition for the theory $(L, S)$ to be true. That is, the two conceptions meaning and truth are completely intertwined.

\footnotetext{
${ }^{3}$ I am grateful to Márton Gömöri for calling my attention to this issue.
} 
4. As semantics, in the above holistic sense, is a part and parcel of physical theory, no physical theory without semantics. Therefore, in case of empirical failure of a physical theory, semantics is one of the possible subjects of revision. In other words, semantics is as much hypothetical as any other part of the theory. For example, given that $L$ is consistent, one can easily see that the following statements cannot hold true at the same time:

$$
A \text { refers to } a
$$

$$
L \vdash A
$$

$$
a \text { is not the case in } U
$$

since (i) and (iii) would imply $L \vdash \neg A$. Therefore, observing that $a$ is not the case we are not entitled to say that we observe that " $\neg A$ ". Simply because if $a$ is not the case, then condition (b) fails, the whole semantics is lost. Therefore $\neg A$ does not carry meaning at all. That is to say, the state of affairs when $a$ is not the case is something unexpressed, a brute phenomenon. This sheds light on the constitutive role of semantics, or rather, of the whole theory, in the sense of Reichenbach's "constitutive $a$ priori" (Reichenbach 1965).

10. Thus, as we discussed in point $\mathbf{8}$, condition (b) requires a real correlation between physical facts of two parts of the physical world, $L$ and $U$. Combining this with the thesis of

$$
\text { the causal closeness of the physical world, and }
$$

$$
\text { the principle of common cause } 4 \text {, }
$$

one must conclude that both semantic relationship and the truth of the physical theory (consequently, our knowledge) must be brought about by the underlying causal processes of the physical world, going on in the common causal past of the two parts of the world $L$ and $U$. This underlying process is what we normally call learning through experience. This is a very strong support of empiricism. No knowledge of the physical world is possible without experience. By the same token, no semantically meaningful talk about the physical world is possible without experience. There is no a priori meaning and there is no $a$ priori truth.

\footnotetext{
${ }^{4}$ I mean the Reichenbachian thesis that no correlation without causation; every correlation is either due to a direct causal effect, or is brought about by a third factor, a so-called common cause (e.g. Reichenbach 1956; Hofer-Szabó et al. 2013).
} 
In agreement with what was said in point 4 , it is worthwhile mentioning that the same is true with respect to the meta-mathematical theories. According to the physico-formalist claims, a formal system is a particular part of the physical world. Any theory describing a formal system, therefore, describes a part of the physical world; that is, it is a particular case of physical theory. There is no a priori meaning and truth in a meta-mathematical theory either.

11. It is a deep belief of many physicists that mathematics provides us the key ideas that are realized in the natural world. In Einstein's words:

Our experience up to date justifies us in feeling sure that in Nature is actualized the ideal of mathematical simplicity. It is my conviction that pure mathematical construction enables us to discover the concepts and the laws connecting them which give us the key to the understanding of the phenomena of Nature. Experience can of course guide us in our choice of serviceable mathematical concepts; it cannot possibly be the source from which they are derived; experience of course remains the sole criterion of the serviceability of a mathematical construction for physics, but the truly creative principle resides in mathematics. In a certain sense, therefore, I hold it to be true that pure thought is competent to comprehend the real, as the ancients dreamed. (Einstein 1934, 167)

One might raise the question, if mathematics is only about the formal systems without meaning, how it is, then, possible that mathematical structures prove themselves to be so expressive in the physical applications. As Richard Feynman put it: "I find it quite amazing that it is possible to predict what will happen by mathematics, which is simply following rules which really have nothing to do with the original thing." (Feynman 1967, 171)

Let me start with mentioning that it is not mathematics alone by which the physicist can predict what will happen, but physical axioms and mathematics together. The physical axioms are determined by empirical facts. More exactly, the physicist, keeping, as long as possible, the logical and mathematical axioms fixed, tunes the physical axioms such that the theorems derivable from the unified system of logical, mathematical, and physical axioms be compatible with the empirical facts. Consequently, the employed logical and mathematical structures in themselves need not reflect anything about the real world in order to be useful.

Let me explain this with an analogy. You can experience a similar situation when you change the mouse driver on your computer (or just change the mouse 
settings): first you feel that the pointer movements ("derived theorems") generated by the new driver ("mathematics") according to your previously habituated hand movements ("physical axioms") do not faithfully reflect the geometry of your screen. Then, keeping the driver (and driver settings) fixed, you tune your hand movements - through typical "trial and error" learning - such that the generated pointer movements fit to the arrangement of your screen content.

Thus, there is no miraculous "preadaption" involved just because certain aspects of empirical reality "fit themselves into the forms provided by mathematics". This is simply a result of selections made by the physicist. Just as there is no preadaption at work when you successfully can install kitchen units obtained from a department store in your kitchen. The rules according to which the shelves, cupboards and doors can be combined show nothing about the actual geometry of your kitchen. But the final result is that the kitchen "fits itself" to the form of the whole set, as if through a kind of preadaption.

12. The constitutive role of formal systems in our physical knowledge by no means entitles us to say that there is a hypostatized a priori conceptual scheme in terms of which we grasp the experienced physical reality, and that this conceptual scheme generates analytic truths. For, what there is is anything but not conceptual: we only have the flesh and blood formal systems without any reference or meaning. Once an otherwise meaningless formula of a formal system is provided with meaning, in the sense of point 7 , it becomes true or false in a non-analytic sense.

\section{Acknowledgment}

The research was partly supported by the (Hungarian) National Research, Development and Innovation Office, No. K100715 and No. K115593.

\section{References}

Ayer, Alfred J. 1952. Language,Truth and Logic. New York: Dover Publications.

Colyvan, Mark. 2000. "Conceptual contingency and abstract existence." The Philosophical Quarterly 50: 87-91.

Colyvan, Mark. 2004. "Indispensability Arguments in the Philosophy of Mathematics." The Stanford Encyclopedia of Philosophy (Fall 2004 Edition), Edward N. Zalta, ed. 
Crossley, J.N., C.J. Ash, J.C. Stillwell, N.H. Williams, and C.J. Brickhill. 1990. What is mathematical logic? New York: Dover Publications.

Curry, Haskell B. 1951. Outlines of a Formalist Philosophy of Mathematics. Amsterdam: North-Holland.

Einstein, Albert. 1934. "On the Method of Theoretical Physics." Philosophy of Science 1: 163-169.

Feynman, Richard. 1967. The character of physical law. Cambridge: MIT Press.

Field, Hartry. 1993. "The Conceptual Contingency of Mathematical Objects." Mind 102: 285-299.

Hofer-Szabó, Gábor, Miklós Rédei, and László E. Szabó. 2013. The Principle of the Common Cause. Cambridge: Cambridge University Press.

Reichenbach, Hans. 1956. The Direction of Time. Berkeley: University of California Press.

Reichenbach, Hans. 1965. The Theory of Relativity and A Priori Knowledge (Berkeley: University of California Press).

Miller, Kristie. 2012. „Mathematical Contingentism.” Erkenntnis 77: 335-359.

Szabó, László E. 2003. "Formal System as Physical Objects: A Physicalist Account of Mathematical Truth." International Studies in the Philosophy of Science 17: 117-125.

Szabó, László E. 2012. "Mathematical facts in a physicalist ontology." Parallel Processing Letters 22: 1240009. 